\title{
Ellipse chains inscribed inside a parabola and integer sequences
}

\section{Giovanni Lucca}

\author{
Piacenza, Italy \\ vanni_lucca@inwind.it \\ Submitted: July 11, 2020 \\ Accepted: September 25, 2020 \\ Published online: September 26, 2020
}

\begin{abstract}
The paper presents formulas and conditions relevant to the construction of chains of mutually tangent ellipses inscribed inside a parabola. Moreover, some connections with certain integer sequences and Pythagorean triplets are shown.
\end{abstract}

Keywords: Ellipse chains, parabola, integer sequences, Pythagorean triplets. MSC: 51M04, 51M15

\section{Introduction}

In the previous paper [2], we studied the problem of inscribing a chain of mutually tangent circles inside a parabola; here we want to generalise it by considering the case of ellipses instead of circles.

We also mention that a cognate problem has been presented in [1] by considering a hyperbola instead of a parabola.

Let us consider a parabola in its simplest form that is:

$$
y=a x^{2}, \quad a>0 .
$$

This is not a limitation because, as known, the shape of the parabola depends only on the coefficient of the second order term; moreover, the main results presented in this paper do not change in the case when $a<0$. The advantage in considering only the case $a>0$ consists in obtaining simpler formulas. 
Inside this parabola, we want to inscribe an infinite chain of ellipses where the generic $i$-th ellipse is tangent to the preceding and succeeding ones; see an example in Figure 1.

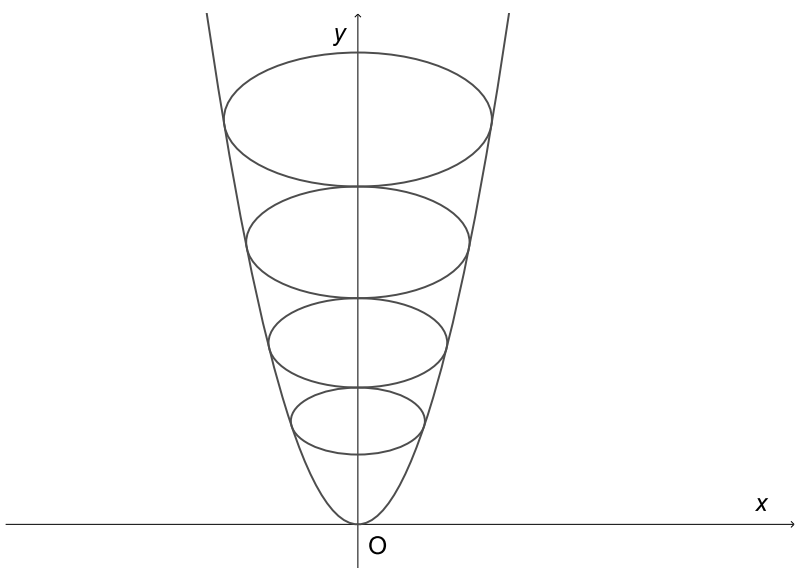

Figure 1: Example of ellipse chain inscribed inside a parabola

\section{Construction of the ellipse chain}

For symmetry reasons, the centre of each ellipse must be placed on the ordinate axis; thus, the centre of the generic $i$-th ellipse of the chain has coordinates $\left(0, Y_{i}\right)$.

Moreover, we define respectively by $\alpha_{i}$ and $\beta_{i}$ the horizontal and vertical semiaxes of the generic $i$-th ellipse.

In the next subsections, we introduce the hypotheses adopted and the basic conditions needed to build up the ellipse chain.

\subsection{Similarity of the ellipses}

The first basic assumption we make is that all the ellipses forming the chain are similar that is

$$
\lambda=\frac{\alpha_{i}}{\beta_{i}}, \quad \lambda \in \mathbb{R}^{+}, i=0,1, \ldots
$$

Note that it could be $\lambda<1$; in that case, the major axis of the ellipses of the chain is the vertical one.

\subsection{Tangency condition between to consecutive ellipses}

By considering two consecutive ellipses of the chain, we have that the difference between the ordinate centres is equal to the sum of the vertical semi-axis that is

$$
Y_{i}-Y_{i-1}=\beta_{i}+\beta_{i-1}, \quad i=1,2, \ldots
$$




\subsection{Tangency condition between parabola and ellipses}

In order to find the intersections between the parabola and the generic $i$-th ellipse, we have to consider the following equation system

$$
\left\{\begin{array}{l}
y=a x^{2}, \\
\frac{x^{2}}{\alpha_{i}^{2}}+\frac{\left(y-Y_{i}\right)^{2}}{\beta_{i}^{2}}=1 .
\end{array}\right.
$$

By solving with respect to $y$, one obtains

$$
y=\frac{-\beta_{i}^{2}+2 a \alpha_{i}^{2} Y_{i} \pm \beta_{i} \sqrt{\beta_{i}^{2}-4 a \alpha_{i}^{2} Y_{i}+4 a^{2} \alpha_{i}^{4}}}{2 a \alpha_{i}^{2}} .
$$

In order that the ellipses of the chain are tangent to the parabola, we have, from equation (2.3), that the discriminant $\Delta=\beta_{i}^{2}-4 a \alpha_{i}^{2} Y_{i}+4 a^{2} \alpha_{i}^{4}$ must be zero; therefore the tangency condition is

$$
\beta_{i}^{2}-4 a \alpha_{i}^{2} Y_{i}+4 a^{2} \alpha_{i}^{4}=0
$$

\subsection{Condition relating $\lambda, a$ and $\boldsymbol{\beta}_{0}$}

Even if we are considering only the case with $a>0$, it is necessary to remark that by looking at equation (2.3), one has that the sign of the ordinates $y_{T i}$ of the tangency points (just given by equation (2.3) when equation (2.4) holds) between ellipses and parabola must be consistent with the sign of $a$; i.e., they must be positive when $a$ is positive and vice-versa. Therefore, we must have

$$
\left\{\begin{array}{l}
y_{T i}=\frac{-\beta_{i}^{2}+2 a \alpha_{i}^{2} Y_{i}}{2 a \alpha_{i}^{2}} \geq 0 \text { if } a>0 \\
y_{T i}=\frac{-\beta_{i}^{2}+2 a \alpha_{i}^{2} Y_{i}}{2 a \alpha_{i}^{2}} \leq 0 \text { if } a<0
\end{array}\right.
$$

In the case when $a>0$, equation (2.5) is verified if

$$
Y_{i} \geq \frac{1}{2 a \lambda^{2}}, \quad i=0,1, \ldots
$$

Clearly, if the following relationship holds

$$
Y_{0} \geq \frac{1}{2 a \lambda^{2}}
$$

then also (2.6) is verified because the relation $Y_{i} \geq Y_{0}$ is always fullfilled. Neverthless, it must also be $Y_{0} \geq \beta_{0}$ because, in order to have no intersections between the first ellipse and the parabola, the ordinate of the centre of the first ellipse cannot be smaller than its vertical semi-axis length; so, we can write the following relation

$$
\min \left(Y_{0}\right)=\beta_{0} .
$$


Thus, by considering the case $Y_{0}=\beta_{0}$, from relation (2.7) we finally obtain

$$
\frac{1}{\beta_{0} a \lambda^{2}} \leq 2
$$

Condition (2.8) or equivalently

$$
\frac{1}{\alpha_{0} a \lambda} \leq 2
$$

are the basic relationships, relating the parameters of the parabola and of the ellipse chain, that must be fulfilled in order to be able to construct the ellipse chain itself.

\subsection{Recursive formulas}

Let us consider equation (2.4); by means of (2.1) it can be written as

$$
\beta_{i}^{2}-4 a \lambda^{2} \beta_{i}^{2} Y_{i}+4 a^{2} \lambda^{4} \beta_{i}^{4}=0 .
$$

Being $\beta_{i} \neq 0$, it can be simplified into

$$
1-4 a \lambda^{2} Y_{i}+4 a^{2} \lambda^{4} \beta_{i}^{2}=0 .
$$

We also have

$$
1-4 a \lambda^{2} Y_{i-1}+4 a^{2} \lambda^{4} \beta_{i-1}^{2}=0 .
$$

By subtracting the corresponding members of the two above equations, by means of equation (2.2) one gets

$$
\beta_{i}=\beta_{i-1}+\frac{1}{a \lambda^{2}}, \quad i=1,2, \ldots
$$

By substituting (2.9) into (2.2) one finally has

$$
Y_{i}=Y_{i-1}+2 \beta_{i-1}+\frac{1}{a \lambda^{2}}, \quad i=1,2, \ldots
$$

Equation (2.9) together equation (2.10) form a system of non homogeneous linear recursive relations that allow us to built the ellipse chain starting from the pair of initial values $\left(\beta_{0}, Y_{0}\right)$ where $\beta_{0}$ must full-fill relation (2.8) and $Y_{0}$ is given by

$$
Y_{0}=a \lambda^{2} \beta_{0}^{2}+\frac{1}{4 a \lambda^{2}}
$$

as one can deduce from (2.4) when $i=0$.

Clearly, the values of $\alpha_{i}$ can be determined by remembering (2.1). 


\section{Some integer sequences associated to the ellipse chains}

In this paragraph, we focus our attention on the particular chains characterised by the following relationship

$$
Y_{0}=\beta_{0} .
$$

All these chains have in common the characteristic that the first ellipse is tangent to the parabola at its vertex (see Figure 2).

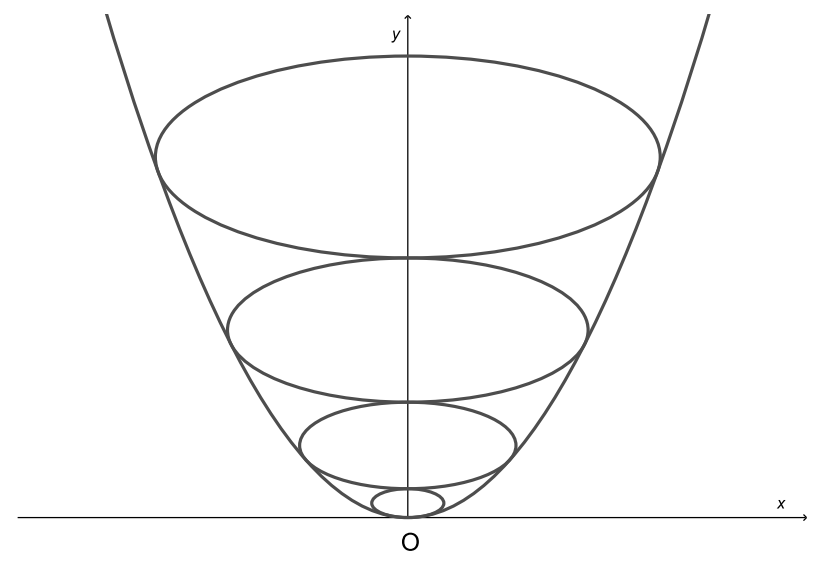

Figure 2: Example of ellipse chain with tangency point at the parabola vertex

Remark 3.1. In this case we have that

$$
\frac{1}{\beta_{0} a \lambda^{2}}=2
$$

This kind of ellipse chains, as we shall see in the following, are in relation with certain integer sequences that do not depend neither on $a$, that is the shape of the parabola, nor on $\lambda$, that is the ratio between the ellipse semi-axes, but, on the contrary, they can be considered as common and invariant sequences to be related to the set of all parabolas with inscribed ellipse chains disposed as in Figure 2.

Let us introduce the following sequences $\left\{\bar{Y}_{i}\right\},\left\{\bar{\alpha}_{i}\right\},\left\{\bar{\beta}_{i}\right\}$ respectively defined as

$$
\bar{Y}_{i}=\frac{Y_{i}}{Y_{0}}, \quad \bar{\alpha}_{i}=\frac{\alpha_{i}}{\alpha_{0}}, \quad \bar{\beta}_{i}=\frac{\beta_{i}}{\beta_{0}} .
$$

Remark 3.2. By remembering equation (2.1) and from the definitions of $\left\{\bar{\alpha}_{i}\right\}$ and $\left\{\bar{\beta}_{i}\right\}$, one has:

$$
\left\{\bar{\alpha}_{i}\right\}=\left\{\bar{\beta}_{i}\right\} .
$$

Thus, in the following, we focus only on sequence $\left\{\bar{\beta}_{i}\right\}$. 
We now derive some theorems related to the above introduced sequences.

Theorem 3.3. Sequence $\left\{\bar{\beta}_{i}\right\}$ is the sequence of the odd numbers.

Proof. By dividing both the members of equation (2.12) by $\beta_{0}$ and by taking into account equation (3.2) one gets

$$
\bar{\beta}_{i}=\bar{\beta}_{i-1}+2, \quad i=1,2, \ldots
$$

By remembering that $\bar{\beta}_{0}=1$, from equation (3.4), it follows, by induction, that $\left\{\bar{\beta}_{i}\right\}$ is the sequence of the odd numbers.

Sequence $\left\{\bar{\beta}_{i}\right\}$ is classified in the On-Line Encyclopedia of Integer Sequences OEIS [3] as A005408.

As far as sequence $\left\{\bar{Y}_{i}\right\}$ is concerned, the following theorem holds:

Theorem 3.4. Sequence $\left\{\bar{Y}_{i}\right\}$ is the integer sequence $\left\{2 i^{2}+2 i+1\right\}$.

Proof. From equations (2.4) and (2.1) one obtains

$$
Y_{i}=a \lambda^{2} \beta_{i}^{2}+\frac{1}{4 a \lambda^{2}}
$$

By dividing both the members of equation (3.5) by $\beta_{0}$ and by taking into account of equations (3.1) and (3.2) and of Theorem 3.3 one has

$$
\bar{Y}_{i}=\frac{1}{2}(2 i+1)^{2}+\frac{1}{2}=2 i^{2}+2 i+1, \quad i=0,1, \ldots
$$

which was to be proved.

This sequence is classified in OEIS as A046092.

Let us consider now, the ordinates of the tangency points $y_{T i}$ of the ellipses to the parabola given by equation (2.5). From this equation, we have that $y_{T i}$ is given by

$$
y_{T i}=Y_{i}-\frac{1}{2 a \lambda^{2}}, \quad i=1,2, \ldots
$$

Then, we can define a further sequence $\left\{\bar{y}_{T i}\right\}$ as follows

$$
\bar{y}_{T i}=\frac{y_{T i}}{\beta_{0}}, \quad i=1,2, \ldots
$$

and the following theorem holds:

Theorem 3.5. Sequence $\left\{\bar{y}_{T i}\right\}$ is the integer sequence $\left\{2 i^{2}+2 i\right\}$.

Proof. From equations (3.7) and (3.8) we have

$$
\bar{y}_{T i}=\frac{Y_{i}}{\beta_{0}}-\frac{1}{2 \beta_{0} a \lambda^{2}}, \quad i=1,2, \ldots
$$

By remembering equations (3.6) and (3.2), one finally has:

$$
\bar{y}_{T i}=2 i^{2}+2 i, \quad i=1,2, \ldots
$$

which was to be proved. 
The sequence $\left\{\bar{y}_{T i}\right\}$ can be found in OEIS as well. It is classified as: A001844.

If we consider the area $A_{i}$ of the $i$-th ellipse, it is given by

$$
A_{i}=\pi \alpha_{i} \beta_{i}
$$

Thus, we can introduce another sequence $\left\{\bar{A}_{i}\right\}$ defined as

$$
\bar{A}_{i}=\frac{A_{i}}{A_{0}}, \quad i=0,1, \ldots
$$

By considering this sequence, we have the following theorem:

Theorem 3.6. The sequence $\left\{\bar{A}_{i}\right\}$ is the integer sequence given by the square of the odd numbers.

Proof. We have that $\bar{A}_{i}$ is given by

$$
\bar{A}_{i}=\frac{\alpha_{i}}{\alpha_{0}} \frac{\beta_{i}}{\beta_{0}}, \quad i=0,1, \ldots
$$

and from Theorem 3.3 and equation (3.3) it follows that

$$
\bar{A}_{i}=(2 i+1)^{2}, \quad i=0,1, \ldots
$$

which was to be proved.

This sequence is classified in OEIS as A016754.

The results here found, relevant to the integer sequences, are consistent with the ones appearing in [2] which are a particular case of the work here presented when $\alpha_{i}=\beta_{i}$, i.e., the ellipses degenerate into circles.

\section{Relation with Pythagorean triplets}

By looking at the sequences $\left\{\bar{\beta}_{i}\right\},\left\{\bar{y}_{T i}\right\}$ and $\left\{\bar{Y}_{i}\right\}$ for $i=1,2, \ldots$, they have a particular characteristic that puts them in relation with the primitive Pythagorean triplets.

In fact, the following theorem holds:

Theorem 4.1. The sequences $\left\{\bar{\beta}_{i}\right\},\left\{\bar{y}_{T i}\right\}$ and $\left\{\bar{Y}_{i}\right\}$ for $i=1,2, \ldots$ form an infinite set of primitive Pythagorean triplets.

Proof. By remembering that $\beta_{i}=2 i+1$ and by using equations (3.6) and (3.10), one can immediately verify that:

$$
\bar{\beta}_{i}^{2}+\bar{y}_{T i}^{2}=\bar{Y}_{i}^{2}, \quad i=1,2, \ldots
$$

so meaning that the corresponding terms of these sequences form a Pythagorean triplet; in particular, these Pythagorean triplets are also primitive. 
In fact, we have that, for each $i$ with $(i=1,2, \ldots), \bar{y}_{T i}=\frac{\bar{\beta}_{i}^{2}-1}{2}$ and $\bar{Y}_{i}=\frac{\bar{\beta}_{i}^{2}+1}{2}$. On the other hand, a well known algorithm, attributed to Pythagoras himself, allows to generate a primitive Pythagorean triplet starting from any odd integer number $2 i+1$; according to it, the primitive triplet is given by

$$
\left(2 i+1, \frac{(2 i+1)^{2}-1}{2}, \frac{(2 i+1)^{2}+1}{2}\right) .
$$

Being $\overline{\beta_{i}}$ an odd integer, we have that the triplet

$$
\left(2 i+1, \frac{(2 i+1)^{2}-1}{2}, \frac{(2 i+1)^{2}+1}{2}\right)
$$

is identical to the triplet $\left(\bar{\beta}_{i}, \bar{y}_{T i}, \bar{Y}_{i}\right)$ so deducing that it is primitive.

Remark 4.2. Notice that for $i=1$, the corresponding first three terms of the three above sequences form the basic primitive Pythagorean triplet $(3,4,5)$.

Acknowledgements. The author would like to thank the anonymous reviewer for the useful comments and suggestions that allowed to improve the paper.

\section{References}

[1] H. Belbachir, L. Németh, S. M. Tebtoub: Integer sequences and ellipse chains inside a hyperbola, Annales Mathematicae et Informaticae 52 (2020), DOI: https://doi.org/10.33039/ami.2020.06.002.

[2] G. LuccA: Integer sequences, Pythagorean triplets and circle chains inscribed inside a parabola, International Journal of Geometry 8.1 (2019), pp. 22-31.

[3] N. J. A. Sloane: The On-Line Encyclopedia of Integer Sequences, URL: https://oeis.org. 